\title{
Long Baseline Navigation with Clock Offset Estimation and Discrete-Time Measurements
}

\author{
Pedro Batista* \\ Institute for Systems and Robotics, Instituto Superior Técnico, Universidade de Lisboa \\ Av. Rovisco Pais, 1049-001 Lisboa, Portugal
}

\begin{abstract}
This paper proposes a novel one-way-travel-time long baseline filtering system that includes the estimation of the clocks' offset, which is assumed constant. Considering discrete-time pseudo-range measurements, in addition to the data provided by a Doppler velocity log and an attitude and heading reference system, an augmented system is derived that can be regarded as linear for observability and observer design purposes. Its observability is discussed and a Kalman filter provides the estimation solution, with globally exponentially stable (GES) error dynamics. Simulation results are presented to evaluate the proposed solution, which is also compared with the EKF, including Monte Carlo runs.
\end{abstract}

Keywords: Navigation, long baseline, pseudo-range, clock offset, estimation.

\section{Introduction}

Whether for geo-referencing or control purposes, navigation data is essential for the successful operation of underwater vehicles. The aim of this paper is to provide an alternative methodology for one-way-travel-time (OWTT) long baseline (LBL) navigation with explicit continuous clock offset dynamic estimation.

In underwater applications the global positioning system (GPS) is unavailable due to the strong attenuation that the electromagnetic field suffers in water. As such, alternative positioning methods are required, see [11] and [20] for early references on underwater acoustic positioning systems. LBL navigation is one of the most popular choices when it comes to underwater vehicles, where the two-way-travel-time of acoustic signals from the vehicle to several transponders is usually used for trilateration. In [28] three acoustic transponders, with known inertial positions, are considered and an extended Kalman filter (EKF) with a Rauch-Tung-Striebel smoother is implemented to obtain filtered estimates of the states. In [32] a conventional LBL positioning system is combined with a Doppler sonar, with bottom-lock, that includes a magnetometer and roll/pitch sensors, and a complementary low-pass/high-pass filter approach is employed to show that the Doppler precision can be improved. Two different LBL approaches are presented in [29]: i) fix computation approach; and ii) filtering approach. In the socalled fix computation approach, dead-reckoning is performed between acoustic fixes, which reset the vehicle position whenever available. In the second approach, dead-reckoning is performed but valid travel times are used, whenever available, to correct for dead-reckoning drift. In [15] preliminary field trials are reported of a navigation system that employs a LBL acoustic

${ }^{*}$ Corresponding author. Fax: +351218418291

Email address: pbatista@isr.ist.utl.pt (Pedro Batista) positioning system, a Doppler sonar, a fiber-optic North seeking gyro, pressure sensors and magnetic compasses, where the main navigation algorithm resorts to the least-squares method.

A different concept than LBL, where one aims to estimate a segment of the trajectory instead of the current position, is proposed in [13], where diffusion-based trajectory observers are considered. In [17] the concept of LBL navigation is extended to the case where measurements to a single acoustic source are available, by combining dead-reckoning and rich trajectories to obtain a so-called synthetic long baseline. A similar approach is proposed in [16], where simulations of a so-called virtual long baseline (VLBL) navigation algorithm for autonomous underwater vehicles are presented. The explicit use of single range measurements without trilateration is evaluated in [9], where the range measurements are fused with the the data obtained from an INS in an EKF. Alternative single range solutions can be found, for instance, in [22], [30], [7], and [3]. Finally, in terms of acoustic-based navigation methods, it is worth mentioning ultra-short baseline (USBL) acoustic positioning systems, which are rapidly increasing in popularity, see e.g. [23], [21], and references therein. For interesting discussions and detailed surveys on underwater vehicle navigation techniques and challenges see [14], [18], and [27], as the literature review provided herein is not exhaustive.

An alternative to two-way-travel-time LBL navigation is OWTT navigation, where synchronous clocks between beacons are assumed available and the time of signal emission is either predefined or encoded and sent through communication modems, see [6], [31], and references therein. However, clock synchronization at the beginning of each mission poses an additional and very heavy burden. Furthermore, clock drift is inevitable in long missions unless synchronization is performed periodically. In previous work by the authors a novel filtering solution was proposed for LBL navigation [4] based on an extension of the framework for single range measurements de- 
tailed in [3]. In these approaches, the range measurements were assumed to be available, either resorting to two-way-travel-time or to OWTT coupled with synchronous clocks and acoustic communications. In short, the system dynamics are augmented, including as system states the range measurements and identifying nonlinear terms with new system states, until the system can be regarded as linear. A careful observability analysis follows and, due to its constructive nature, the Kalman filter provides the estimation solution with globally exponentially stable (GES) error dynamics.

The main contribution of this paper is the development of a novel filtering system for LBL navigation, within a OWTT scheme, that includes the estimation of the unknown offset between the receiver and the emitters clocks, which is assumed constant. As a result of the unknown clock offset, instead of ranges one has pseudo-range measurements, which correspond to the true ranges plus an unknown offset. Low rate discretetime pseudo-range measurements force the filtering error to converge to zero, while higher rate attitude and velocity measurements drive the system dynamics. An augmented system, which can be regarded as linear for observability analysis and observer design purposes, is derived considering the LBL configuration and the introduction of new system states. Its observability is carefully studied and the Kalman filter naturally provides an estimation solution, with GES error dynamics. Previous work by the author on OWTT LBL navigation can be found in [2], where the novel LBL framework with an OWTT scheme was first described. This paper presents the complete analysis of the system, including observability proofs, as well as the evaluation of the estimation performance, with Monte Carlo runs and a comparison with the EKF. Overall, the simulation results presented herein are also more realistic: slower update rates are considered and clock drift is also incorporated. For the latter, the Kalman filter parameters are tunned such that a slowly time-varying clock offset can be successfully tracked. Previous works applying state augmentation to applications with LBL positioning can be found in [19], [24], and [26], where past measurements were included in the state. In the present work the differences between pseudo-ranges are considered as additional states.

Another possible source of error in OWTT LBL navigation is the sound speed profile. That specific problem has been addressed by the author, in a two-way-travel-time setting, in [1]. Addressing both issues, i.e., determining the sound speed and the clock offset in a OWTT setting, is out of the scope of this paper. In underwater acoustic navigation multipath is also a common problem. While that is also out of the scope of the paper, there exist many algorithms in the literature to handle the presence of outliers, see e.g. [22] and [33].

The problem statement and the nominal system dynamics are introduced in Section 2, while the filter design is detailed in Section 3. Simulation results are presented in Section 4 and Section 5 summarizes the main results of the paper.

\subsection{Notation}

Throughout the paper the symbol $\mathbf{0}$ denotes a matrix of zeros and $\mathbf{I}$ an identity matrix, both of appropriate dimensions. A block diagonal matrix is represented by $\operatorname{diag}\left(\mathbf{A}_{1}, \ldots, \mathbf{A}_{n}\right)$. For $\mathbf{x} \in \mathbb{R}^{3}$ and $\mathbf{y} \in \mathbb{R}^{3}, \mathbf{x} \cdot \mathbf{y}$ represents the inner product.

\section{Problem statement}

Consider a LBL acoustic positioning system, consisting of a set of emitters that are fixed in the mission scenario, where an underwater vehicle operates, which is equipped with an acoustic receiver, as depicted in Fig. 1. The inertial positions of the emitters, which are constant, are assumed available to the vehicle. In an OWTT setting, the fixed beacons, which are assumed

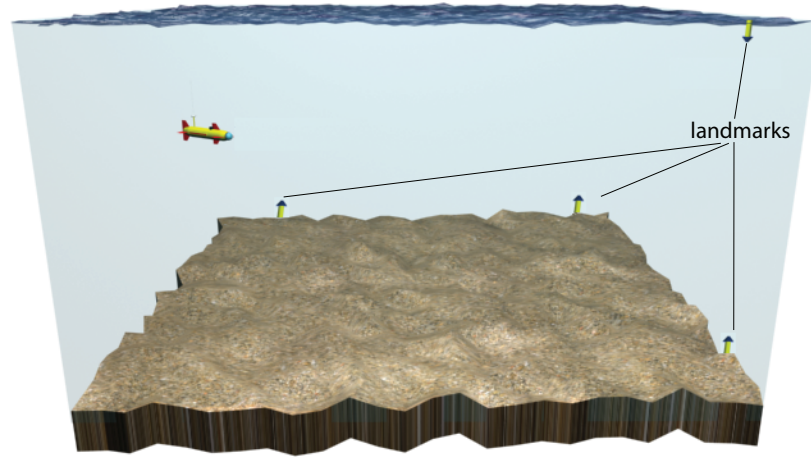

Figure 1: Long baseline mission scenario

to have their clocks synchronized, emit a signal, tagged with the senders' time or at a predefined broadcast time, which is then received by the acoustic receiver that is installed on-board the agent. Typically, the clock of the receiver is also synchronized with that of the receivers and the distance is computed from the time-of-flight and the sound speed profile. In this paper, the offset between the emitting and receiving clocks is assumed to be an unknown constant, and as such instead of range measurements one has pseudo-range measurements, which are measured periodically. Further suppose that the vehicle is equipped with an attitude and heading reference system (AHRS) and a Doppler velocity log (DVL). The problem considered herein is that of designing a continuous-discrete filter, with globally exponentially stable error dynamics, to estimate the position and linear velocity of the vehicle, as well as the offset between the emitting and receiving clocks.

\subsection{System dynamics}

Let $\{I\}$ denote a local inertial reference coordinate frame and $\{B\}$ a coordinate frame attached to the vehicle, usually referred to as the body-fixed reference frame. An example of a local inertial frame is the North-East-Down (NED) frame. Although this is not an inertial frame due to the rotation of the Earth, it can be assumed as such for applications as in this paper. Then, the linear motion of the vehicle is described by

$$
\dot{\mathbf{p}}(t)=\mathbf{R}(t) \mathbf{v}(t),
$$

where $\mathbf{p}(t) \in \mathbb{R}^{3}$ corresponds to the inertial position of the vehicle, $\mathbf{v}(t) \in \mathbb{R}^{3}$ is the velocity of the vehicle relative to $\{I\}$, 
expressed in body-fixed coordinates, and $\mathbf{R}(t) \in S O(3)$ is the rotation matrix from $\{B\}$ to $\{I\}$.

The AHRS provides the rotation matrix $\mathbf{R}(t)$, while the DVL measures, in the absence of bottom-lock, the velocity of the vehicle relative to the fluid, expressed in body-fixed coordinates. Let $\mathbf{v}_{c}(t) \in \mathbb{R}^{3}$ denote the velocity of the fluid, in inertial coordinates, and $\mathbf{v}_{r}(t) \in \mathbb{R}^{3}$ be the DVL reading, i.e., the velocity of the vehicle relative to the fluid, expressed in body-fixed coordinates. Then,

$$
\mathbf{v}(t)=\mathbf{R}^{T}(t) \mathbf{v}_{c}(t)+\mathbf{v}_{r}(t) .
$$

Finally, let $\mathbf{s}_{i} \in \mathbb{R}^{3}, i=1, \ldots, L$, denote the inertial positions of the beacons. Then, the pseudo-range measurements are given by

$$
r_{i}(k)=\left\|\mathbf{s}_{i}-\mathbf{p}\left(t_{k}\right)\right\|+b_{c}\left(t_{k}\right),
$$

with $t_{k}:=t_{0}+k T, k \in \mathbb{N}$, where $T>0$ is the sampling period, $t_{0}$ is the initial time, and $b_{c}\left(t_{k}\right)$ is a bias term that accounts for the effect of the unknown offset of the clocks between the emitters and the receiver. The following assumptions are considered in the paper.

Assumption 1. The pseudo-range measurements are positive, i.e., $r_{i}(k)>0$ for all $k$ and $i=1, \ldots, L$.

Assumption 2. The inertial fluid velocity is constant, i.e.,

$$
\dot{\mathbf{v}}_{c}(t)=\mathbf{0} \text {. }
$$

Assumption 3. The offset of the clocks is constant, i.e.,

$$
\dot{b}_{c}(t)=0 \text {. }
$$

With these assumptions and combining (1)-(3) gives the nonlinear system with discrete outputs

$$
\left\{\begin{array}{l}
\dot{\mathbf{p}}(t)=\mathbf{v}_{c}(t)+\mathbf{R}(t) \mathbf{v}_{r}(t) \\
\dot{\mathbf{v}}_{c}(t)=\mathbf{0} \\
\dot{b}_{c}(t)=0 \\
r_{1}(k)=\left\|\mathbf{s}_{1}-\mathbf{p}\left(t_{k}\right)\right\|+b_{c}\left(t_{k}\right) \\
\vdots \\
r_{L}(k)=\left\|\mathbf{s}_{L}-\mathbf{p}\left(t_{k}\right)\right\|+b_{c}\left(t_{k}\right)
\end{array}\right.
$$

The problem considered in this paper is the design of an estimation solution for (4) with globally exponentially stable error dynamics.

Remark 1. The technical condition stated in Assumption 1 is a mild one, which can always be verified in practice. Indeed, if the time tag of a signal received by the vehicle was greater than the clock of the receiver, the receiver would immediately know that its clock is behind and could reset it to a higher value, so that the pseudo-range measurement is positive.

\section{Filter design}

In previous work by the authors [4] a novel LBL framework was proposed in continuous time. In short, additional states and outputs are derived that allow to consider the system as linear in the state, even though it still is, in fact, nonlinear. This is done by means of identification of some nonlinear terms as new variables and noticing that the output and input are available signals for observer design purposes. In this paper, a similar approach is pursued but considering: i) discrete-time measurements; and ii) pseudo-ranges, with an unknown constant clock offset. This setting leads to a different state vector and consequently a different dynamic system, and captures the nature of the underwater ranging sensing system when the offset between the clocks of the acoustic emitters fixed in the mission scenario and the clock of the acoustic receiver installed on-board the vehicle is unknown but constant.

\subsection{Discretization and system augmentation}

The exact discrete-time system dynamics corresponding to (4) are given by

$$
\left\{\begin{array}{l}
\mathbf{p}\left(t_{k+1}\right)=\mathbf{p}\left(t_{k}\right)+T \mathbf{v}_{c}\left(t_{k}\right)+\int_{t_{k}}^{t_{k+1}} \mathbf{R}(\tau) \mathbf{v}_{r}(\tau) d \tau \\
\mathbf{v}_{c}\left(t_{k+1}\right)=\mathbf{v}_{c}\left(t_{k}\right) \\
b_{c}\left(t_{k+1}\right)=b_{c}\left(t_{k}\right) \\
r_{1}(k)=\left\|\mathbf{s}_{1}-\mathbf{p}\left(t_{k}\right)\right\|+b_{c}\left(t_{k}\right) \\
\vdots \\
r_{L}(k)=\left\|\mathbf{s}_{L}-\mathbf{p}\left(t_{k}\right)\right\|+b_{c}\left(t_{k}\right)
\end{array} .\right.
$$

Define the discrete-time states

$$
\left\{\begin{array}{l}
\mathbf{x}_{1}(k):=\mathbf{p}\left(t_{k}\right) \\
\mathbf{x}_{2}(k):=\mathbf{v}_{c}\left(t_{k}\right) \\
x_{3}(k)=b_{c}\left(t_{k}\right)
\end{array} .\right.
$$

From (5) one may write

$$
\left\{\begin{array}{l}
\mathbf{x}_{1}(k+1)=\mathbf{x}_{1}(k)+T \mathbf{x}_{2}(k)+\mathbf{u}(k) \\
\mathbf{x}_{2}(k+1)=\mathbf{x}_{2}(k) \\
x_{3}(k+1)=x_{3}(k)
\end{array},\right.
$$

with

$$
\mathbf{u}(k):=\int_{t_{k}}^{t_{k+1}} \mathbf{R}(\tau) \mathbf{v}_{r}(\tau) d \tau .
$$

As a function of the system states, the pseudo-ranges can be written as

$$
r_{i}(k)=\left\|\mathbf{s}_{i}-\mathbf{x}_{1}(k)\right\|+x_{3}(k), i=1, \ldots, L .
$$

To encode the LBL structure, take the difference of the squares of pseudo-range measurements to two different beacons, which gives

$$
\begin{gathered}
r_{i}^{2}(k)-r_{j}^{2}(k)=-2\left(\mathbf{s}_{i}-\mathbf{s}_{j}\right) \cdot \mathbf{x}_{1}(k) \\
+2\left[r_{i}(k)-r_{j}(k)\right] x_{3}(k)+\left\|\mathbf{s}_{i}\right\|^{2}-\left\|\mathbf{s}_{j}\right\|^{2}
\end{gathered}
$$

for all $i, j \in\{1, \ldots, L\}, i \neq j$. Using

$$
r_{i}^{2}(k)-r_{j}^{2}(k)=\left[r_{i}(k)+r_{j}(k)\right]\left[r_{i}(k)-r_{j}(k)\right]
$$


in (6) allows to write the difference of the pseudo-ranges to different emitters as

$$
\begin{gathered}
r_{i}(k)-r_{j}(k)=-2 \frac{\mathbf{s}_{i}-\mathbf{s}_{j}}{r_{i}(k)+r_{j}(k)} \cdot \mathbf{x}_{1}(k) \\
+2 \frac{r_{i}(k)-r_{j}(k)}{r_{i}(k)+r_{j}(k)} x_{3}(k)+\frac{\left\|\mathbf{s}_{i}\right\|^{2}-\left\|\mathbf{s}_{j}\right\|^{2}}{r_{i}(k)+r_{j}(k)}
\end{gathered}
$$

for all $i, j \in\{1, \ldots, L\}, i \neq j$. This approach bears some resemblance with the one presented in [5] for the LBL problem, however here the clock offset effect is also included. Consider these differences as new system states, i.e., define

$$
\left\{\begin{array}{l}
x_{4}(k)=r_{1}(k)-r_{2}(k) \\
x_{5}(k)=r_{1}(k)-r_{3}(k) \\
\vdots \\
x_{2+C_{2}^{L}}(k)=r_{L-2}(k)-r_{L}(k) \\
x_{3+C_{2}^{L}}(k)=r_{L-1}(k)-r_{L}(k)
\end{array}\right.
$$

where $C_{2}^{L}$ is the number of 2-combinations of $L$ elements, i.e. $C_{2}^{L}=L(L-1) / 2$. It is a matter of computation (see Appendix A) to show that the dynamics of the new states can be written as

$$
\begin{gathered}
r_{i}(k+1)-r_{j}(k+1)=-2 T \frac{\mathbf{s}_{i}-\mathbf{s}_{j}}{r_{i}(k+1)+r_{j}(k+1)} \cdot \mathbf{x}_{2}(k) \\
+2 \frac{\left[r_{i}(k+1)-r_{i}(k)\right]-\left[r_{j}(k+1)-r_{j}(k)\right]}{r_{i}(k+1)+r_{j}(k+1)} x_{3}(k) \\
+\frac{r_{i}(k)+r_{j}(k)}{r_{i}(k+1)+r_{j}(k+1)}\left[r_{i}(k)-r_{j}(k)\right]-2 \frac{\left(\mathbf{s}_{i}-\mathbf{s}_{j}\right) \cdot \mathbf{u}(k)}{r_{i}(k+1)+r_{j}(k+1)}
\end{gathered}
$$

for all $i, j \in\{1, \ldots, L\}, i \neq j$.

Define the augmented state vector as

$$
\mathbf{x}(k):=\left[\begin{array}{c}
\mathbf{x}_{1}(k) \\
\mathbf{x}_{2}(k) \\
x_{3}(k) \\
x_{4}(k) \\
\vdots \\
x_{3+C_{2}^{L}}(k)
\end{array}\right] \in \mathbb{R}^{3+3+1+C_{2}^{L}}
$$

Then, the discrete-time system dynamics can be written as

$$
\mathbf{x}(k+1)=\mathbf{A}(k) \mathbf{x}(k)+\mathbf{B u}_{a}(k),
$$

where $\mathbf{A}(k) \in \mathbb{R}^{\left(7+C_{2}^{L}\right) \times\left(7+C_{2}^{L}\right)}$,

$$
\mathbf{A}(k)=\left[\begin{array}{ccc|c}
\mathbf{I} & T \mathbf{I} & \mathbf{0} & \mathbf{0} \\
\mathbf{0} & \mathbf{I} & \mathbf{0} & \mathbf{0} \\
\mathbf{0} & \mathbf{0} & 1 & \mathbf{0} \\
\hline \mathbf{0} & \mathbf{A}_{42}(k) & \mathbf{A}_{43}(k) & \mathbf{A}_{44}(k)
\end{array}\right],
$$

with

$$
\mathbf{A}_{42}(k)=-2 T\left[\begin{array}{c}
\frac{\mathbf{s}_{1}^{T}-\mathbf{s}_{2}^{T}}{r_{1}(k+1)+r_{2}(k+1)} \\
\frac{\mathbf{s}_{1}^{T}-\mathbf{s}_{3}^{T}}{r_{1}(k+1)+r_{3}(k+1)} \\
\vdots \\
\frac{\mathbf{s}_{L-2}^{T}-\mathbf{s}_{L}^{T}}{r_{L-2}(k+1)+r_{L}(k+1)} \\
\frac{\mathbf{s}_{L-1}^{T}-\mathbf{s}_{L}^{T}}{r_{L-1}(k+1)+r_{L}(k+1)}
\end{array}\right] \in \mathbb{R}^{C_{2}^{L} \times 3},
$$

$$
\mathbf{A}_{43}(k)=2\left[\begin{array}{c}
\frac{\left[r_{1}(k+1)-r_{1}(k)\right]-\left[r_{2}(k+1)-r_{2}(k)\right]}{r_{1}(k+1)+r_{2}(k+1)} \\
\frac{\left[r_{1}(k+1)-r_{1}(k)\right]-\left[r_{3}(k+1)-r_{3}(k)\right]}{r_{1}(k+1)+r_{3}(k+1)} \\
\vdots \\
\frac{\left[r_{L-2}(k+1)-r_{L-2}(k)\right]-\left[r_{L}(k+1)-r_{L}(k)\right]}{r_{L-2}(k+1)+r_{L}(k+1)} \\
\frac{\left[r_{L-1}(k+1)-r_{L-1}(k)\right]-\left[r_{L}(k+1)-r_{L}(k)\right]}{r_{L-1}(k+1)+r_{L}(k+1)}
\end{array}\right],
$$

$$
\begin{gathered}
\mathbf{A}_{44}(k)=\operatorname{diag}\left(\frac{r_{1}(k)+r_{2}(k)}{r_{1}(k+1)+r_{2}(k+1)}, \frac{r_{1}(k)+r_{3}(k)}{r_{1}(k+1)+r_{3}(k+1)},\right. \\
\left.\ldots, \frac{r_{L-2}(k)+r_{L}(k)}{r_{L-2}(k+1)+r_{L}(k+1)}, \frac{r_{L-1}(k)+r_{L}(k)}{r_{L-1}(k+1)+r_{L}(k+1)}\right), \\
\mathbf{B}=\left[\begin{array}{cc}
\mathbf{I} & \mathbf{0} \\
\mathbf{0} & \mathbf{0} \\
\mathbf{0} & \mathbf{0} \\
\mathbf{0} & \mathbf{I}
\end{array}\right],
\end{gathered}
$$

and

$$
\mathbf{u}_{a}(k)=\left[\begin{array}{c}
\mathbf{u}(k) \\
\hline-2 \frac{\left(\mathbf{s}_{1}-\mathbf{s}_{2}\right) \cdot \mathbf{u}(k)}{r_{1}(k+1)+r_{2}(k+1)} \\
-2 \frac{\left(\mathbf{s}_{1}-\mathbf{s}_{3}\right) \cdot \mathbf{u}(k)}{r_{1}(k+1)+r_{3}(k+1)} \\
\vdots \\
-2 \frac{\left(\mathbf{s}_{L-2}-\mathbf{s}_{L}\right) \cdot \mathbf{u}(k)}{r_{L-2}(k+1)+r_{L}(k+1)} \\
-2 \frac{\left(\mathbf{s}_{L-1}-\mathbf{s}_{L}\right) \cdot \mathbf{u}(k)}{r_{L-1}(k+1)+r_{L}(k+1)}
\end{array}\right] \in \mathbb{R}^{3+C_{2}^{L} .}
$$

In order the define the augmented system, discard the original nonlinear output, consider (7) and notice that the states $x_{4}(k), \ldots, x_{3+C_{2}^{L}}(k)$ are available from the pseudo-range measurements, which gives in compact form

$$
\left\{\begin{array}{l}
\mathbf{x}(k+1)=\mathbf{A}(k) \mathbf{x}(k)+\mathbf{B} \mathbf{u}_{a}(k) \\
\mathbf{y}(k+1)=\mathbf{C}(k+1) \mathbf{x}(k+1)
\end{array},\right.
$$

with

$$
\mathbf{C}(k)=\left[\begin{array}{cccc}
\mathbf{0} & \mathbf{0} & \mathbf{0} & \mathbf{I} \\
\mathbf{C}_{21}(k) & \mathbf{0} & \mathbf{C}_{33}(k) & \mathbf{I}
\end{array}\right] \in \mathbb{R}^{2 C_{2}^{L} \times\left(7+C_{2}^{L}\right)},
$$

$$
\mathbf{C}_{21}(k)=2\left[\begin{array}{c}
\frac{\mathbf{s}_{1}^{T}-\mathbf{s}_{2}^{T}}{r_{1}(k)+r_{2}(k)} \\
\frac{\mathbf{s}_{1}^{T}-\mathbf{s}_{3}^{T}}{r_{1}(k)+r_{3}(k)} \\
\vdots \\
\frac{\mathbf{s}_{L-2}^{T}-\mathbf{s}_{L}^{T}}{r_{L-2}(k)+r_{L}(k)} \\
\frac{\mathbf{s}_{L-1}^{T}-\mathbf{s}_{L}^{T}}{r_{L-1}(k)+r_{L}(k)}
\end{array}\right]
$$

and

$$
\mathbf{C}_{33}(k)=-2\left[\begin{array}{c}
\frac{r_{1}(k)-r_{2}(k)}{r_{1}(k)+r_{2}(k)} \\
\frac{r_{1}(k)-r_{3}(k)}{r_{1}(k)+r_{3}(k)} \\
\vdots \\
\frac{r_{L-2}(k)-r_{L}(k)}{r_{L-2}(k)+r_{L}(k)} \\
\frac{r_{L-1}(k)-r_{L}(k)}{r_{L-1}\left(k+r_{L}(k)\right.}
\end{array}\right]
$$

Remark 2. Notice that the system (9) is well defined under Assumption 1. 


\subsection{Observability analysis}

The discrete time-varying system (9) can be regarded as linear in the state for observer design purposes, even though the system matrices $\mathbf{A}(k)$ and $\mathbf{C}(k)$ depend on the pseudo-range measurements. This is possible because for observer (or filter) design purposes the pseudo-ranges are available and, hence, they can be simply considered as functions of time. Therefore, linear system theory applies.

The following result addresses the observability of the nonlinear discrete-time system (9).

Theorem 1. Suppose that, for some time $k_{i} \geq k_{0}$, the configuration of the long baseline acoustic positioning system is such that

$$
\mathbf{L}\left(k_{i}\right):=\left[\begin{array}{cc}
\left(\mathbf{s}_{1}-\mathbf{s}_{2}\right)^{T} & -\left[r_{1}\left(k_{i}\right)-r_{2}\left(k_{i}\right)\right] \\
\left(\mathbf{s}_{1}-\mathbf{s}_{3}\right)^{T} & -\left[r_{1}\left(k_{i}\right)-r_{3}\left(k_{i}\right)\right] \\
\vdots & \vdots \\
\left(\mathbf{s}_{L-2}-\mathbf{s}_{L}\right)^{T} & -\left[r_{L-2}\left(k_{i}\right)-r_{L}\left(k_{i}\right)\right] \\
\left(\mathbf{s}_{L-1}-\mathbf{s}_{L}\right)^{T} & -\left[r_{L-1}\left(k_{i}\right)-r_{L}\left(k_{i}\right)\right]
\end{array}\right] \in \mathbb{R}^{C_{2}^{L} \times 4}
$$

is full rank, i.e.,

$$
\operatorname{rank}\left(\mathbf{L}\left(\mathrm{k}_{\mathrm{i}}\right)\right)=4
$$

Then, the discrete-time system (9) is observable on $\left[k_{i}, k_{i}+2\right]$ in the sense that the initial state $\mathbf{x}\left(k_{i}\right)$ is uniquely determined by the input $\left\{\mathbf{u}(k): k=k_{i}, k_{i}+1\right\}$ and the output $\left\{\mathbf{y}(k): k=k_{i}, k_{i}+1\right\}$.

Proof. The proof reduces to show that the observability matrix $\boldsymbol{O}\left(k_{i}, k_{i}+2\right)$ associated with the pair $(\mathbf{A}(k), \mathbf{C}(k))$ on $\left[k_{i}, k_{i}+2\right], k_{i} \geq k_{0}$, has rank equal to the number of states of the system. It follows by contradiction. Fix $k_{i}>k_{0}$ and suppose that the rank of the observability matrix is less than the number of states of the system. Then, there exists a unit vector $\mathbf{d}=\left[\begin{array}{llll}\mathbf{d}_{1}^{T} & \mathbf{d}_{2}^{T} & d_{3} & \mathbf{d}_{4}^{T}\end{array}\right]^{T} \in \mathbb{R}^{3+3+1+C_{2}^{L}}$, with $\mathbf{d}_{1}, \mathbf{d}_{2} \in \mathbb{R}^{3}$, $d_{3} \in \mathbb{R}, \mathbf{d}_{4} \in \mathbb{R}^{C_{2}^{L}}$, such that $\boldsymbol{O}\left(k_{i}, k_{i}+2\right) \mathbf{d}=\mathbf{0}$ or, equivalently,

$$
\left\{\begin{array}{l}
\mathbf{C}\left(k_{i}\right) \mathbf{d}=\mathbf{0} \\
\mathbf{C}\left(k_{i}+1\right) \mathbf{A}\left(k_{i}\right) \mathbf{d}=\mathbf{0}
\end{array} .\right.
$$

From the first equation of (11), and attending to the structure of $\mathbf{C}\left(k_{i}\right)$, one immediately concludes that $\mathbf{d}_{4}=\mathbf{0}$. Substituting that in the first equation of (11) gives

$$
\left\{\begin{array}{l}
2 \frac{\left(\mathbf{s}_{1}-\mathbf{s}_{2}\right)^{T} \mathbf{d}_{1}-\left[r_{1}\left(k_{i}\right)-r_{2}\left(k_{i}\right)\right] d_{3}}{r_{1}\left(k_{i}\right)+r_{2}\left(k_{i}\right)}=0 \\
2 \frac{\left(\mathbf{s}_{1}-\mathbf{s}_{3}\right)^{T} \mathbf{d}_{1}-\left[r_{1}\left(k_{i}\right)-r_{3}\left(k_{i}\right)\right] d_{3}}{r_{1}\left(k_{i}\right)+r_{3}\left(k_{i}\right)}=0 \\
\vdots \\
2 \frac{\left(\mathbf{s}_{L-2}-\mathbf{s}_{L}\right)^{T} \mathbf{d}_{1}-\left[r_{L-2}\left(k_{i}\right)-r_{L}\left(k_{i}\right)\right] d_{3}}{r_{L-2}\left(k_{i}\right)+r_{L}\left(k_{i}\right)}=0 \\
2 \frac{\left(\mathbf{s}_{L-1}-\mathbf{s}_{L}\right)^{T} \mathbf{d}_{1}-\left[r_{L-1}\left(k_{i}\right)-r_{L}\left(k_{i}\right)\right] d_{3}}{r_{L-1}\left(k_{i}\right)+r_{L}\left(k_{i}\right)}=0
\end{array}\right.
$$

If (10) holds, then the only solution of (12) is $\mathbf{d}_{1}=\mathbf{0}$ and $d_{3}=0$. Now, with $\mathbf{d}_{1}=\mathbf{0}, d_{3}=0$, and $\mathbf{d}_{4}=\mathbf{0}$, one may write, from the second equation of (11), that

$$
\mathbf{A}_{42}(k) \mathbf{d}_{2}=\mathbf{0}
$$

or, equivalently,

$$
\left\{\begin{array}{c}
-2 T \frac{\left(\mathbf{s}_{1}-\mathbf{s}_{2}\right)^{T} \mathbf{d}_{2}}{r_{1}\left(k_{i}+1\right)+r_{2}\left(k_{i}+1\right)}=0 \\
-2 T \frac{\left(\mathbf{s}_{1}-\mathbf{s}_{3}\right)^{T} \mathbf{d}_{2}}{r_{1}\left(k_{i}+1\right)+r_{3}\left(k_{i}+1\right)}=0 \\
\vdots \\
-2 T \frac{\left(\mathbf{s}_{L-2}-\mathbf{s}_{L}\right)^{T} \mathbf{d}_{2}}{r_{L-2}\left(k_{i}+1\right)+r_{L}\left(k_{i}+1\right)}= \\
-2 T \frac{\left(\mathbf{s}_{L-1}-\mathbf{s}_{L}\right)^{T} \mathbf{d}_{2}}{r_{L-1}\left(k_{i}+1\right)+r_{L}\left(k_{i}+1\right)}=0
\end{array} .\right.
$$

Again, if (10) holds, then the only solution of (13) is $\mathbf{d}_{2}=\mathbf{0}$. But this contradicts the hypothesis of existence of a unit vector d such that (11) holds. Hence, the observability matrix must have rank equal to the number of states of the system, which concludes the proof.

Finally, it is important to stress that, in the definition of the augmented system (9), the original nonlinear outputs $r_{i}(k)=$ $\left\|\mathbf{s}_{i}-\mathbf{x}_{1}(k+1)\right\|+x_{3}(k), i=1, \ldots, L$, were discarded. Furthermore, there is nothing in (9) imposing the nonlinear constraints on the augmented states relating them with the original system states. As such, care must be taken when extrapolating conclusions from the observability of (9) to the observability of (5). The following theorem addresses this issue and provides the means for design of a state observer or filter for (5), as it will be seen afterwards.

Theorem 2. Suppose that (10) holds for some $k_{i} \geq k_{0}$. Then:

i) the nonlinear system (5) is observable on the interval $\left[k_{i}, k_{i}+2\right]$ in the sense that the initial state $\mathbf{x}\left(k_{i}\right)$ is uniquely determined by the input $\left\{\mathbf{u}(k): k=k_{i}, k_{i}+1\right\}$ and the output $\left\{r_{1}(k), \ldots, r_{L}(k): k=k_{i}, k_{i}+1\right\}$; and

ii) the initial condition of the augmented system (9) matches that of (5) on the interval $\left[k_{i}, k_{i}+2\right]$, i.e.,

$$
\left\{\begin{array}{l}
\mathbf{x}_{1}\left(k_{i}\right)=\mathbf{p}\left(t_{k_{i}}\right) \\
\mathbf{x}_{2}\left(k_{i}\right)=\mathbf{v}_{c}\left(t_{k_{i}}\right) \\
x_{3}\left(k_{i}\right)=b_{c}\left(t_{k_{i}}\right) \\
x_{4}\left(k_{i}\right)=\left\|\mathbf{s}_{1}-\mathbf{p}\left(t_{k_{i}}\right)\right\|-\left\|\mathbf{s}_{2}-\mathbf{p}\left(t_{k_{i}}\right)\right\| \\
x_{5}\left(k_{i}\right)=\left\|\mathbf{s}_{1}-\mathbf{p}\left(t_{k_{i}}\right)\right\|-\left\|\mathbf{s}_{3}-\mathbf{p}\left(t_{k_{i}}\right)\right\| \\
\vdots \\
x_{2+C_{2}^{L}}\left(k_{i}\right)=\left\|\mathbf{s}_{L-2}-\mathbf{p}\left(t_{k_{i}}\right)\right\|-\left\|\mathbf{s}_{L}-\mathbf{p}\left(t_{k_{i}}\right)\right\| \\
x_{3+C_{2}^{L}}\left(k_{i}\right)=\left\|\mathbf{s}_{L-1}-\mathbf{p}\left(t_{k_{i}}\right)\right\|-\left\|\mathbf{s}_{L}-\mathbf{p}\left(t_{k_{i}}\right)\right\|
\end{array} .\right.
$$

Proof. Let

$$
\mathbf{x}\left(k_{i}\right):=\left[\begin{array}{c}
\mathbf{x}_{1}\left(k_{i}\right) \\
\mathbf{x}_{2}\left(k_{i}\right) \\
x_{3}\left(k_{i}\right) \\
x_{4}\left(k_{i}\right) \\
\vdots \\
x_{3+C_{2}^{L}}\left(k_{i}\right)
\end{array}\right] \in \mathbb{R}^{3+3+1+C_{2}^{L}}
$$

be the initial condition of (9) and let $\mathbf{p}\left(t_{k_{i}}\right), \mathbf{v}_{c}\left(t_{k_{i}}\right)$, and $b_{c}\left(t_{k_{i}}\right)$ be the initial condition of (5). From the first $C_{2}^{L}$ outputs of (9) 
it must be

$$
\left\{\begin{array}{l}
x_{4}\left(k_{i}\right)=r_{1}\left(k_{i}\right)-r_{2}\left(k_{i}\right) \\
x_{5}\left(k_{i}\right)=r_{1}\left(k_{i}\right)-r_{3}\left(k_{i}\right) \\
\vdots \\
x_{2+C_{2}^{L}}\left(k_{i}\right)=r_{L-2}\left(k_{i}\right)-r_{L}\left(k_{i}\right) \\
x_{3+C_{2}^{L}}\left(k_{i}\right)=r_{L-1}\left(k_{i}\right)-r_{L}\left(k_{i}\right)
\end{array}\right.
$$

or, equivalently,

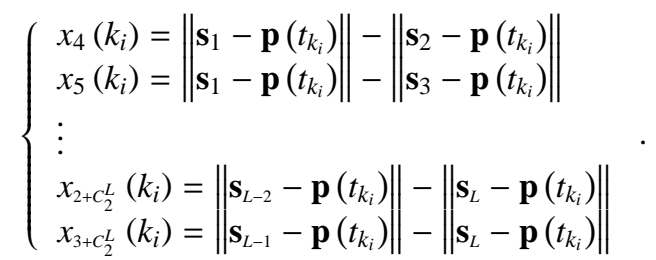

Considering the differences of pseudo-range measurements of the nonlinear system (5) for $k=k_{i}$ as a function of its initial state yields

$$
\begin{gathered}
r_{l}\left(k_{i}\right)-r_{m}\left(k_{i}\right)=-2 \frac{\mathbf{s}_{l}-\mathbf{s}_{m}}{r_{l}\left(k_{i}\right)+r_{m}\left(k_{i}\right)} \cdot \mathbf{p}\left(t_{k_{i}}\right) \\
+2 \frac{r_{l}\left(k_{i}\right)-r_{m}\left(k_{i}\right)}{r_{l}\left(k_{i}\right)+r_{m}\left(k_{i}\right)} b_{c}\left(t_{k_{i}}\right)+\frac{\left\|\mathbf{s}_{l}\right\|^{2}-\left\|\mathbf{s}_{m}\right\|^{2}}{r_{l}\left(k_{i}\right)+r_{m}\left(k_{i}\right)}
\end{gathered}
$$

for all $l, m \in\{1, \ldots, L\}, l \neq m$. On the other hand, evaluating the outputs of (9) $y_{C_{2}^{L}+1}(k)$ to $y_{2 C_{2}^{L}}(k)$ for $k=k_{i}$ as a function of $\mathbf{x}\left(k_{i}\right)$, and considering (14), gives

$$
\begin{gathered}
r_{l}\left(k_{i}\right)-r_{m}\left(k_{i}\right)=-2 \frac{\mathbf{s}_{l}-\mathbf{s}_{m}}{r_{l}\left(k_{i}\right)+r_{m}\left(k_{i}\right)} \cdot \mathbf{x}_{1}\left(k_{i}\right) \\
+2 \frac{r_{l}\left(k_{i}\right)-r_{m}\left(k_{i}\right)}{r_{l}\left(k_{i}\right)+r_{m}\left(k_{i}\right)} x_{3}\left(k_{i}\right)+\frac{\left\|\mathbf{s}_{l}\right\|^{2}-\left\|\mathbf{s}_{m}\right\|^{2}}{r_{l}\left(k_{i}\right)+r_{m}\left(k_{i}\right)}
\end{gathered}
$$

for all $l, m \in\{1, \ldots, L\}, l \neq m$. Comparing (15) with (16), and considering (10), implies that

$$
\left\{\begin{array}{l}
\mathbf{x}_{1}\left(k_{i}\right)=\mathbf{p}\left(t_{k_{i}}\right) \\
x_{3}\left(k_{i}\right)=b_{c}\left(t_{k_{i}}\right)
\end{array} .\right.
$$

For $k=k_{i}+1$ it is possible to write the differences of pseudoranges as a function of the initial state of (5) as

$$
\begin{gathered}
r_{l}\left(k_{i}+1\right)-r_{m}\left(k_{i}+1\right)=\frac{\left\|\mathbf{s}_{l}\right\|^{2}-\left\|\mathbf{s}_{m}\right\|^{2}}{r_{l}\left(k_{i}+1\right)+r_{m}\left(k_{i}+1\right)} \\
-2 \frac{\mathbf{s}_{l}-\mathbf{s}_{m}}{r_{l}\left(k_{i}+1\right)+r_{m}\left(k_{i}+1\right)} \cdot\left[\mathbf{p}\left(t_{k_{i}}\right)+T \mathbf{v}_{c}\left(t_{k_{i}}\right)+\mathbf{u}\left(k_{i}\right)\right] \\
+2 \frac{r_{l}\left(k_{i}+1\right)-r_{m}\left(k_{i}+1\right)}{r_{l}\left(k_{i}+1\right)+r_{m}\left(k_{i}+1\right)} b_{c}\left(t_{k_{i}}\right)
\end{gathered}
$$

for all $l, m \in\{1, \ldots, L\}, l \neq m$. From the first $L$ outputs of (9) for $k=k_{i}+1$ it follows that

$$
\begin{gathered}
r_{l}\left(k_{i}+1\right)-r_{m}\left(k_{i}+1\right)=-2 T \frac{\mathbf{s}_{l}-\mathbf{s}_{m}}{r_{l}\left(k_{i}+1\right)+r_{m}\left(k_{i}+1\right)} \cdot \mathbf{x}_{2}\left(k_{i}\right) \\
+2 \frac{r_{l}\left(k_{i}+1\right)-r_{m}\left(k_{i}+1\right)}{r_{l}\left(k_{i}+1\right)+r_{m}\left(k_{i}+1\right)} x_{3}\left(k_{i}\right)-2 \frac{r_{l}\left(k_{i}\right)-r_{m}\left(k_{i}\right)}{r_{l}\left(k_{i}+1\right)+r_{m}\left(k_{i}+1\right)} x_{3}\left(k_{i}\right) \\
+\frac{r_{l}\left(k_{i}\right)+r_{m}\left(k_{i}\right)}{r_{l}\left(k_{i}+1\right)+r_{m}\left(k_{i}+1\right)}\left[x_{3+l}\left(k_{i}\right)-x_{3+m}\left(k_{i}\right)\right] \\
-2 \frac{\mathbf{s}_{l}-\mathbf{s}_{m}}{r_{l}\left(k_{i}+1\right)+r_{m}\left(k_{i}+1\right)} \cdot \mathbf{u}\left(k_{i}\right)
\end{gathered}
$$

for all $l, m \in\{1, \ldots, L\}, l \neq m$. Using (14) and (17), it is possible to further write

$$
\begin{gathered}
r_{l}\left(k_{i}+1\right)-r_{m}\left(k_{i}+1\right)=-2 T \frac{\mathbf{s}_{l}-\mathbf{s}_{m}}{r_{l}\left(k_{i}+1\right)+r_{m}\left(k_{i}+1\right)} \cdot \mathbf{x}_{2}\left(k_{i}\right) \\
+2 \frac{r_{l}\left(k_{i}+1\right)-r_{m}\left(k_{i}+1\right)}{r_{l}\left(k_{i}+1\right)+r_{m}\left(k_{i}+1\right)} b_{c}\left(t_{k_{i}}\right)-2 \frac{r_{l}\left(k_{i}\right)-r_{m}\left(k_{i}\right)}{r_{l}\left(k_{i}+1\right)+r_{m}\left(k_{i}+1\right)} b_{c}\left(t_{k_{i}}\right) \\
+\frac{r_{l}\left(k_{i}\right)+r_{m}\left(k_{i}\right)}{r_{l}\left(k_{i}+1\right)+r_{m}\left(k_{i}+1\right)}\left[r_{l}\left(k_{i}\right)-r_{m}\left(k_{i}\right)\right] \\
\quad-2 \frac{\mathbf{s}_{l}-\mathbf{s}_{m}}{r_{l}\left(k_{i}+1\right)+r_{m}\left(k_{i}+1\right)} \cdot \mathbf{u}\left(k_{i}\right)
\end{gathered}
$$

for all $l, m \in\{1, \ldots, L\}, l \neq m$. Substituting (16) in (19) and using (17) again gives

$$
\begin{gathered}
r_{l}\left(k_{i}+1\right)-r_{m}\left(k_{i}+1\right)=\frac{\left\|\mathbf{s}_{l}\right\|^{2}-\left\|\mathbf{s}_{m}\right\|^{2}}{r_{l}\left(k_{i}+1\right)+r_{m}\left(k_{i}+1\right)} \\
-2 \frac{\mathbf{s}_{l}-\mathbf{s}_{m}}{r_{l}\left(k_{i}+1\right)+r_{m}\left(k_{i}+1\right)} \cdot\left[\mathbf{p}\left(t_{k_{i}}\right)+T \mathbf{x}_{2}\left(k_{i}\right)+\mathbf{u}\left(k_{i}\right)\right] \\
+2 \frac{r_{l}\left(k_{i}+1\right)-r_{m}\left(k_{i}+1\right)}{r_{l}\left(k_{i}+1\right)+r_{m}\left(k_{i}+1\right)} b_{c}\left(t_{k_{i}}\right)
\end{gathered}
$$

for all $l, m \in\{1, \ldots, L\}, l \neq m$. Comparing (18) with (20) gives

$$
2 T \frac{\mathbf{s}_{l}-\mathbf{s}_{m}}{r_{l}\left(k_{i}+1\right)+r_{m}\left(k_{i}+1\right)} \cdot\left[\mathbf{x}_{2}\left(k_{i}\right)-\mathbf{v}_{c}\left(t_{k_{i}}\right)\right]=0
$$

for all $l, m \in\{1, \ldots, L\}, l \neq m$. If (10) holds, then the only solution of $(21)$ is

$$
\mathbf{x}_{2}=\mathbf{v}_{c}\left(t_{k_{i}}\right) .
$$

This concludes the second part of the theorem, as it has been shown that, in the conditions of the theorem, the initial condition of (5) corresponds to that of (9). Now, notice that, using Theorem 1, the initial condition of (9) is uniquely determined. As, in addition, the two initial conditions match, it follows that the initial condition of (5) is also uniquely determined.

Remark 3. In the setup considered in the paper no pressure/depth sensor is assumed to be installed on-board. Nevertheless, the inclusion of such a sensor is easy and one just needs to consider an additional scalar output, which corresponds to the third coordinate of the position of the vehicle. As a result, the observability conditions are slightly less demanding but also more intricate: on one hand, the minimum number of beacons that are required drops by one, to four beacons; on the other hand, the conditions are slightly more intricate as there is a directionality question involved, as a consequence of the fact that the z-coordinate is rendered observable by the depth sensor.

\subsection{Estimation solution}

The means to design an observer for (5) are provided by Theorem 2 as it is shown that an observer for (9), which can be regarded as linear for observer design purposes, suffices. A simple Kalman filter can be applied, yielding globally exponentially stable error dynamics if the system is shown to be uniformly completely observable [12]. In the paper, the pair $(\mathbf{A}(k), \mathbf{C}(k))$ was shown to be observable. The proof of uniform complete observability follows similar steps considering uniform bounds in time. An alternative to the Kalman filter could be the design of a Luenberger observer as detailed in [25, Theorem 29.2], which would allow to choose the convergence rate.

Notice that, even though the ocean current velocity and the factor that accounts for the unknown clock offset are assumed constant, in nominal terms, by appropriate tuning of the Kalman filter it is possible to successfully track slowly time-varying quantities. An example is provided in the simulations for the case of the clock offset. It is important to stress that, for the case of ocean currents, it is the ocean current that the vehicle experiences that matters. Thus, from the vehicle's perspective, the ocean current might change fast even if at each fixed inertial 
position the ocean current profile is only slowly time-varying. This is because the vehicle may translate through ocean currents which change spatially. For the particular treatment of ocean currents see e.g. [8] and [10].

An observer (or filter) for the discrete-time system (9), as previously derived, only provides estimates when there are range measurements. However, the relative velocity and attitude measurements are usually available at a much higher rate than the range readings. As such, it is possible to obtain estimates of the position, velocity, and clock offset, at a higher rate, using open-loop propagation between range measurements, as given by

$$
\left\{\begin{array}{l}
\hat{\mathbf{x}}_{1}(t)=\hat{\mathbf{x}}_{1}\left(t_{k}\right)+\left(t-t_{k}\right) \hat{\mathbf{x}}_{2}\left(t_{k}\right)+\int_{t_{k}}^{t} \mathbf{R}(\tau) \mathbf{v}_{r}(\tau) d \tau \\
\hat{\mathbf{x}}_{2}(t)=\hat{\mathbf{x}}_{2}\left(t_{k}\right) \\
\hat{x}_{3}(t)=\hat{x}_{3}\left(t_{k}\right)
\end{array}\right.
$$

for $t_{k}<t<t_{k+1}$.

\section{Simulation results}

In order to evaluate the performance of the proposed solution the author performed numerical simulations. The initial position of the vehicle is $\mathbf{p}(0)=\left[\begin{array}{lll}0 & 0 & 10\end{array}\right]^{T} \mathrm{~m}$, while the ocean current velocity was set to $\mathbf{v}_{c}(t)=\left[\begin{array}{lll}-0.1 & 0.20\end{array}\right]^{T} \mathrm{~m} / \mathrm{s}$. The trajectory that was described by the vehicle is shown in Fig. 2. In the simulations presented herein, the LBL configu-

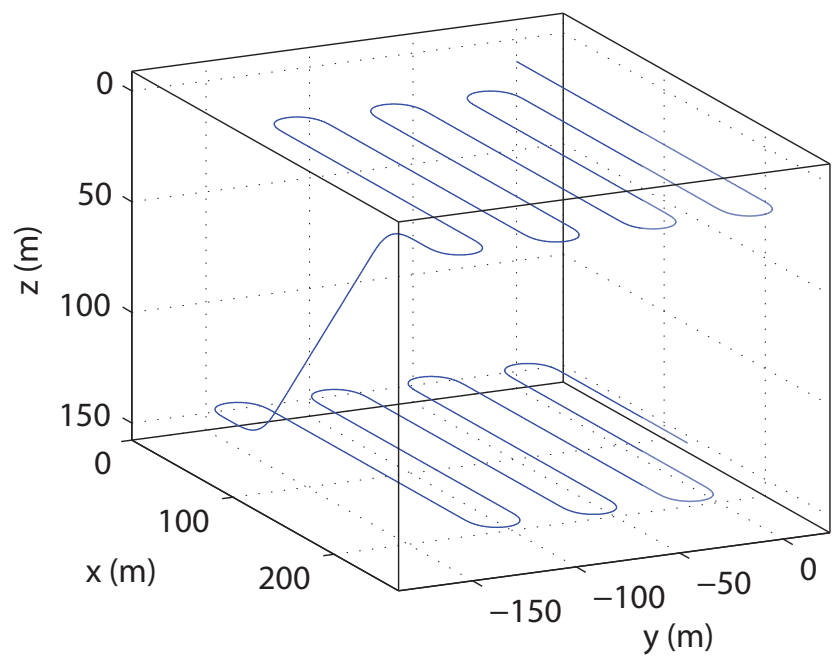

Figure 2: Trajectory described by the vehicle

ration is composed of 5 acoustic beacons and their inertial positions are $\mathbf{s}_{1}=\left[\begin{array}{lll}0 & 0 & 0\end{array}\right]^{T}(\mathrm{~m}), \mathbf{s}_{2}=\left[\begin{array}{lll}0 & 0 & 500\end{array}\right](\mathrm{m})$, $\mathbf{s}_{3}=\left[\begin{array}{lll}500 & 0 & 500\end{array}\right](\mathrm{m}), \mathbf{s}_{4}=\left[\begin{array}{lll}1000 & 0 & 500\end{array}\right](\mathrm{m})$, and $\mathbf{s}_{5}=\left[\begin{array}{lll}0 & 750 & 500\end{array}\right](\mathrm{m})$, so that the rank condition (10) is satisfied. Additional simulations that are not detailed due to space limitations were done, e.g. with a larger network corresponding to multiplying by a factor of 10 each beacon position, and similar results were obtained. The term that accounts for the clocks' offset was set to $b_{c}=50 \mathrm{~m}$.
Sensor noise was considered for all sensors. In particular, the LBL range measurements and the DVL relative velocity readings were assumed to be corrupted by additive uncorrelated zero-mean white Gaussian noise, with standard deviations of $1 \mathrm{~m}$ and $0.01 \mathrm{~m} / \mathrm{s}$, respectively. The attitude, provided by the AHRS and parameterized by roll, pitch, and yaw Euler angles, was also assumed to be corrupted by zero-mean, additive white Gaussian noise, with standard deviation of $0.03^{\circ}$ for the roll and pitch and $0.3^{\circ}$ for the yaw. The sampling period for the range measurements was set to $T=10 \mathrm{~s}$, while the remaining sensors were sampled at $5 \mathrm{~Hz}$. The discrete-time input $\mathbf{u}(k)$, corresponding to a definite integral, was approximated using the trapezoid rule, while the open-loop solution of the position and ocean current velocity estimates, between range measurements, was computed using the Euler method. In fact, as it also corresponds to a definite integral, it is equivalent to the application of the trapezoid rule. Notice that a simplification is considered in these simulations: the pseudo-range measurements are assumed to be available all at the same time and the displacement of the vehicle between range measurements is ignored. If this simplification is not considered, additional computations would be required, as in other LBL solutions, to account for these effects. Finally, multipath was not considered in the simulations as that specific topic is out of the scope of the paper, so no outliers are present. In practice, these can be dealt with one of the methods available in the literature. The inclusion of the pseudo-ranges in the state can be a significant advantage as an outlier rejection algorithm may work at the pseudo-range level instead of working with positions. In a traditional approach, if a position obtained from a set of pseudo-range measurements is considered as an outlier, all those pseudo-range measurements are discarded. However, by working at the pseudo-range level, some pseudo-range measurements may still be employed for update purposes even if others are considered as outliers, thus increasing the amount of information used for estimation purposes.

\subsection{Proposed solution}

To tune the Kalman filter, the state disturbance covariance matrix was chosen as diag $\left(10^{-2} \mathbf{I}, 10^{-6} \mathbf{I}, 10^{-1}, 10^{-6} \mathbf{I}\right)$ and the output noise covariance matrix was set to diag $(0.1 \mathbf{I}, \mathbf{I})$. The initial condition for the position was set with a large initial error, $\hat{\mathbf{x}}_{1}(0)=[200200200]^{T}(\mathrm{~m})$, while the initial estimates of both the ocean current velocity and term that accounts for the offset between the clocks were set to zero. The initial estimates of the pseudo-range differences were set according to the first measurement set that was obtained.

The initial convergence of the position and velocity errors is depicted in Figs. 3(a) and 4(a), whereas the initial convergence of the clock offset error is depicted in Fig. 5(a). The discrete-time updates are visible in these figures, as well as the open-loop propagation intervals. In the latter, both the inertial current velocity error and the clock offset error remain constant, as they are both modeled as constants, while the position error grows linearly (approximately, due to noise) as a result of the ocean current velocity error. As it is possible to observe, the error converges very fast to a neighborhood of zero (due to the 


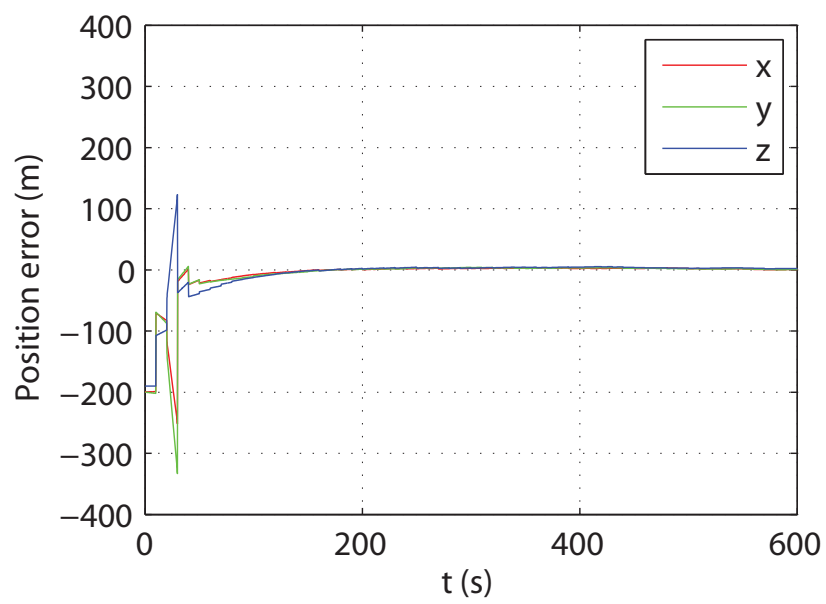

(a) Proposed solution

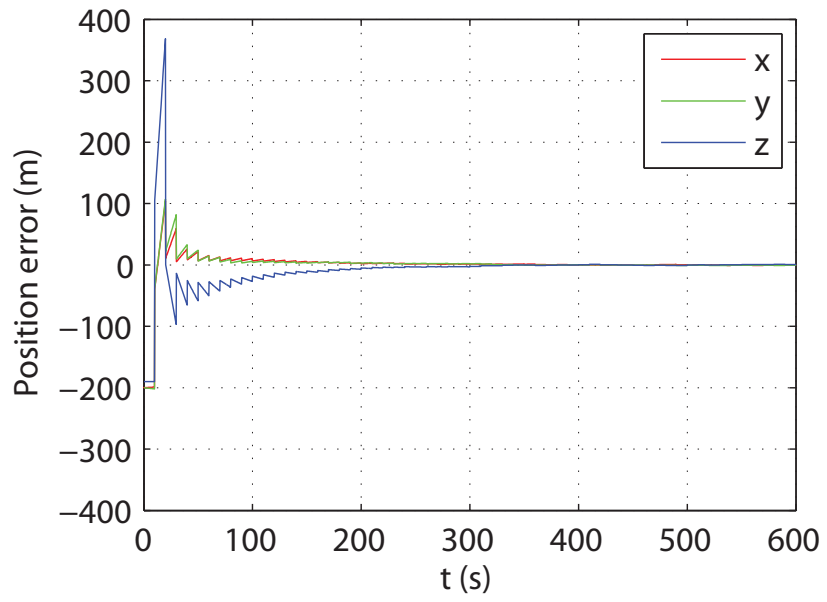

(b) Extended Kalman filter

Figure 3: Initial convergence of the position error

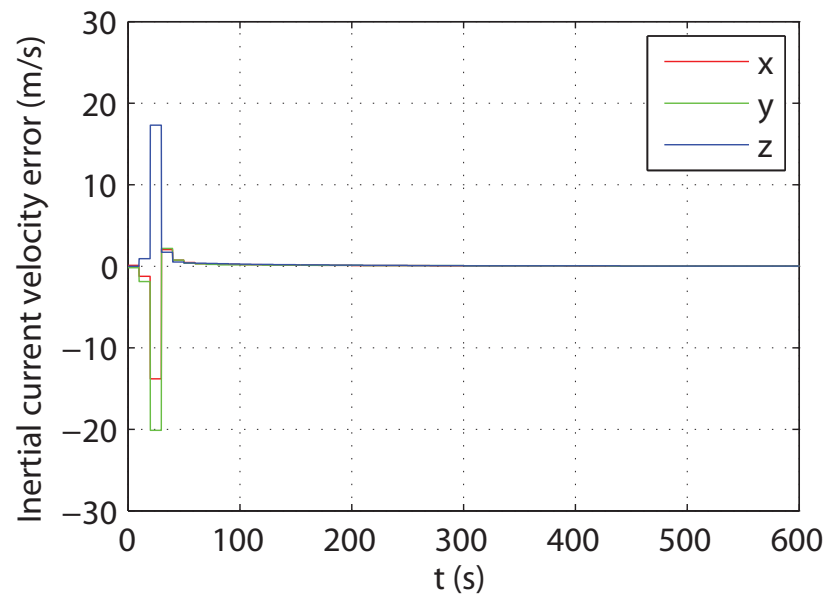

(a) Proposed solution

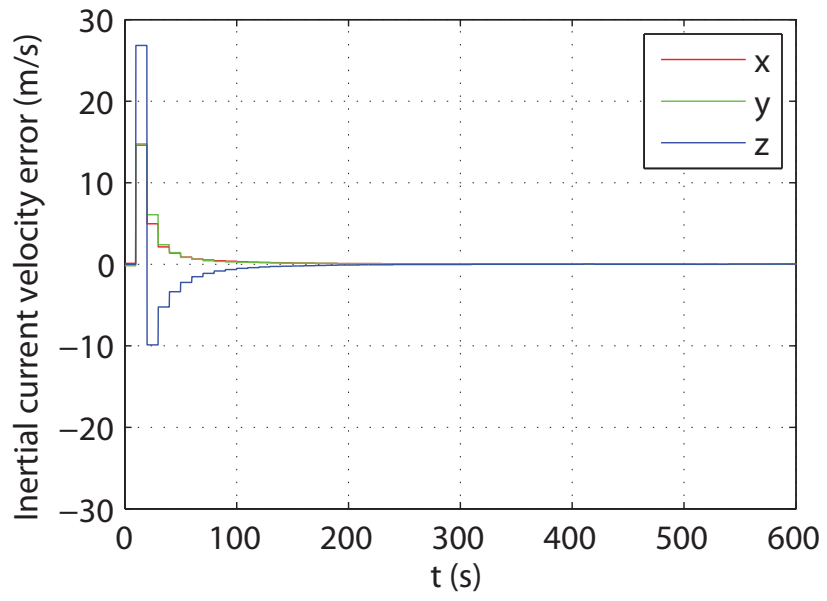

(b) Extended Kalman filter

Figure 4: Initial convergence of the ocean current velocity error

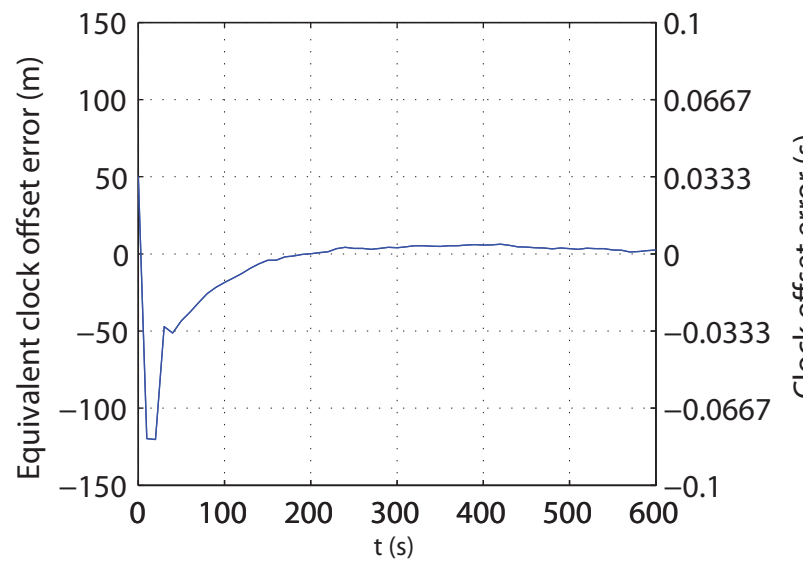

(a) Proposed solution

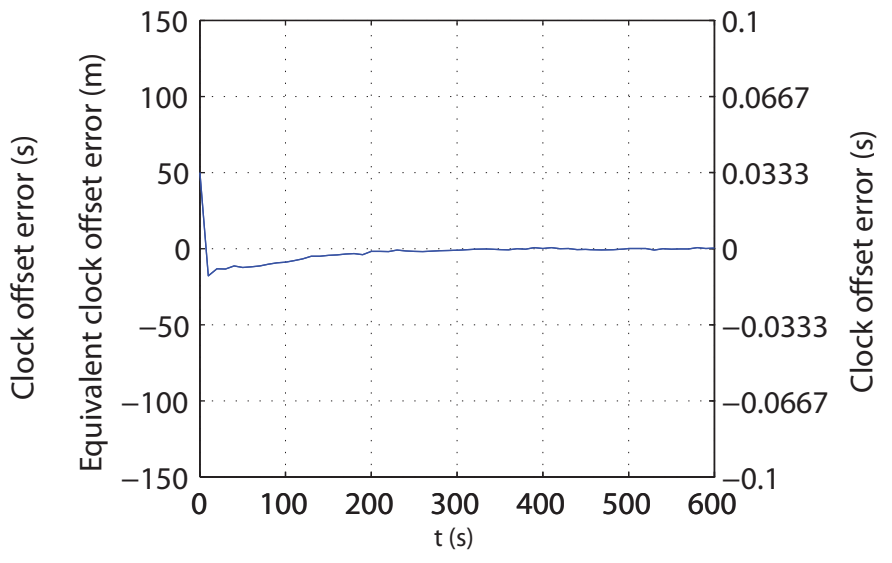

(b) Extended Kalman filter

Figure 5: Initial convergence of the clock offset error 


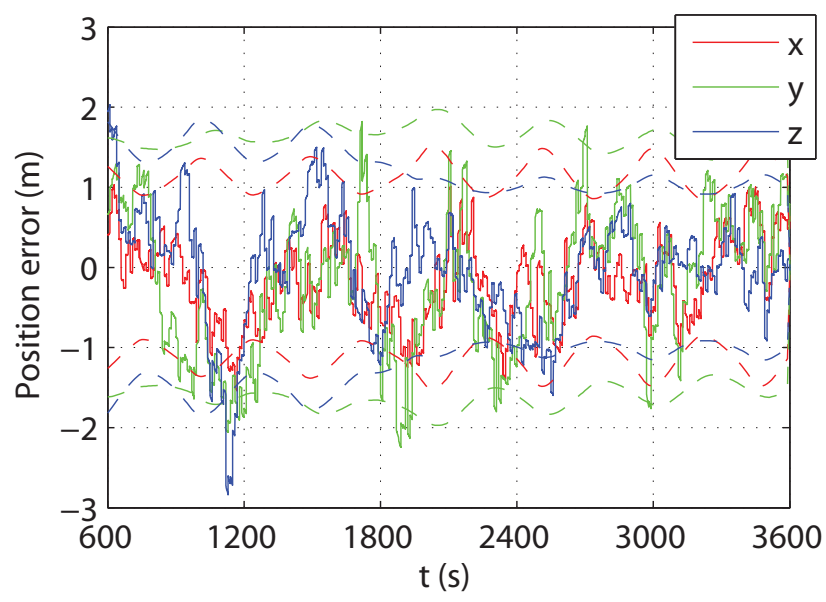

(a) Proposed solution

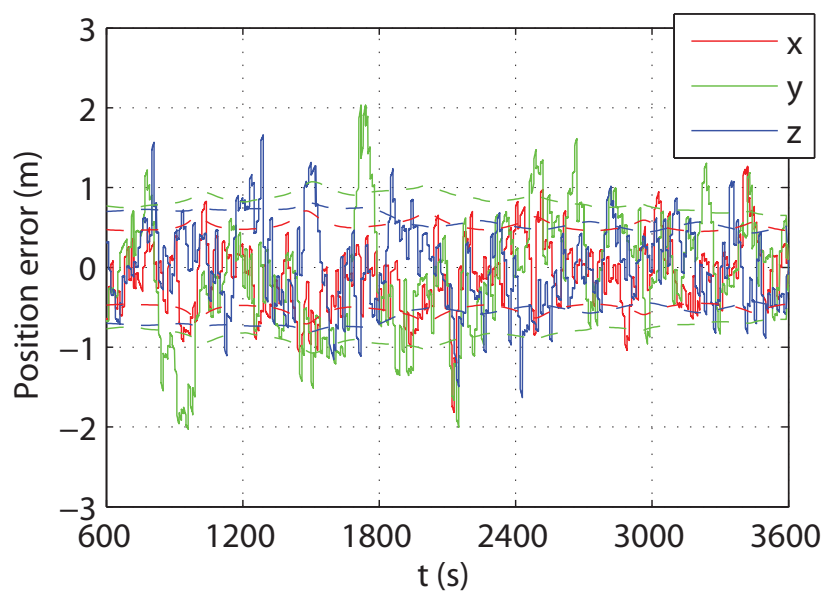

(b) Extended Kalman filter

Figure 6: Steady-state evolution of the position error, with $3-\sigma$ bounds in dashed lines

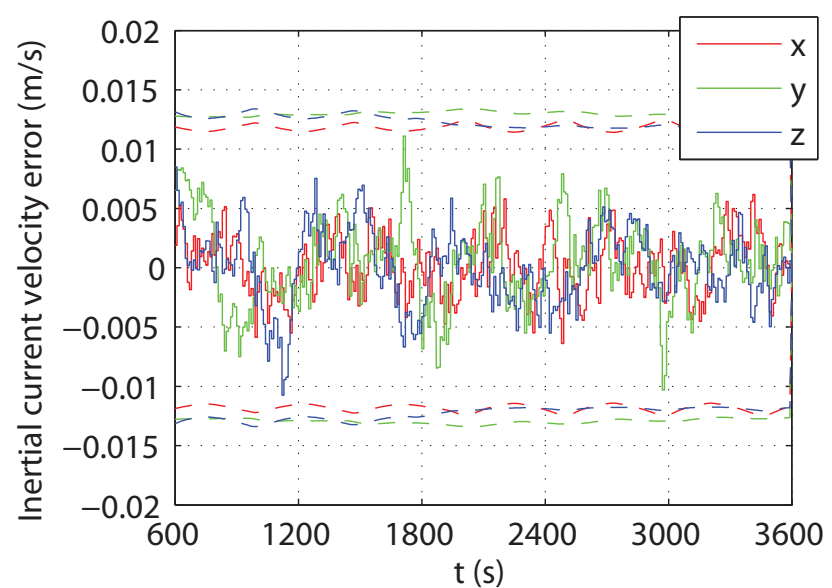

(a) Proposed solution

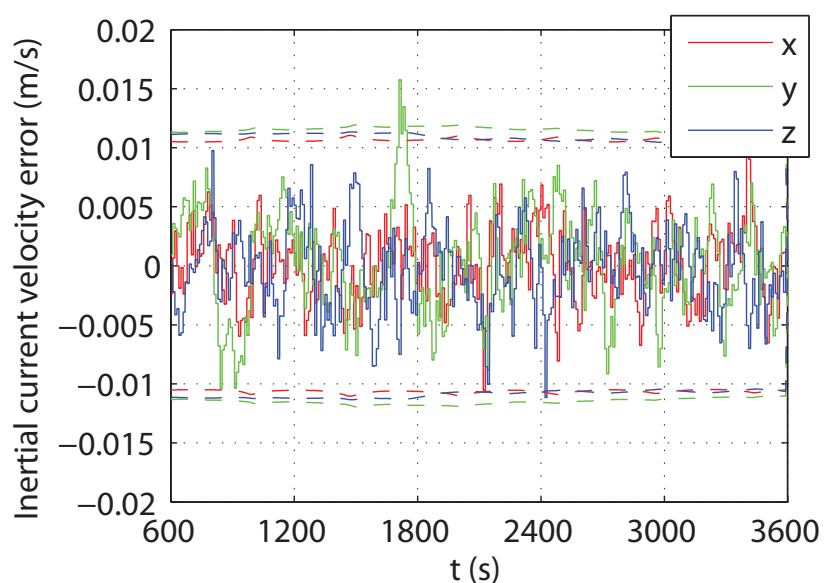

(b) Extended Kalman filter

Figure 7: Steady-state evolution of the ocean current velocity error, with $3-\sigma$ bounds in dashed lines

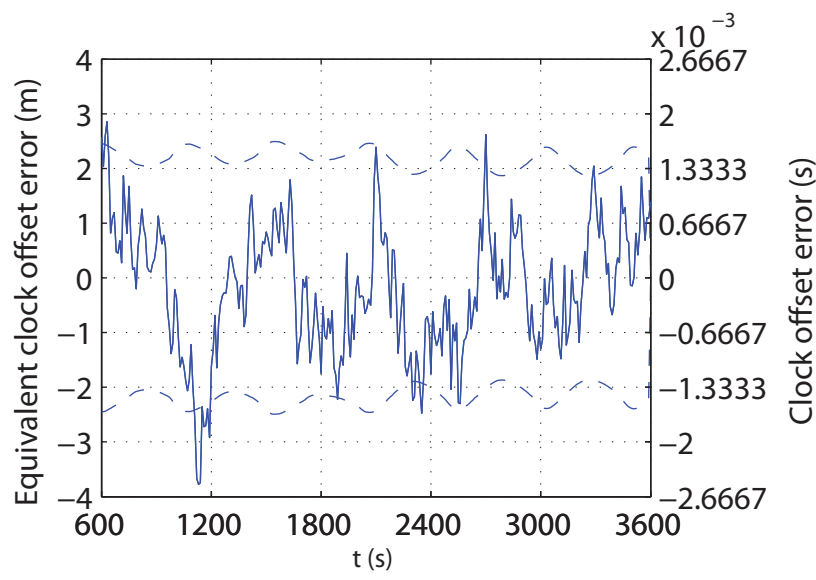

(a) Proposed solution

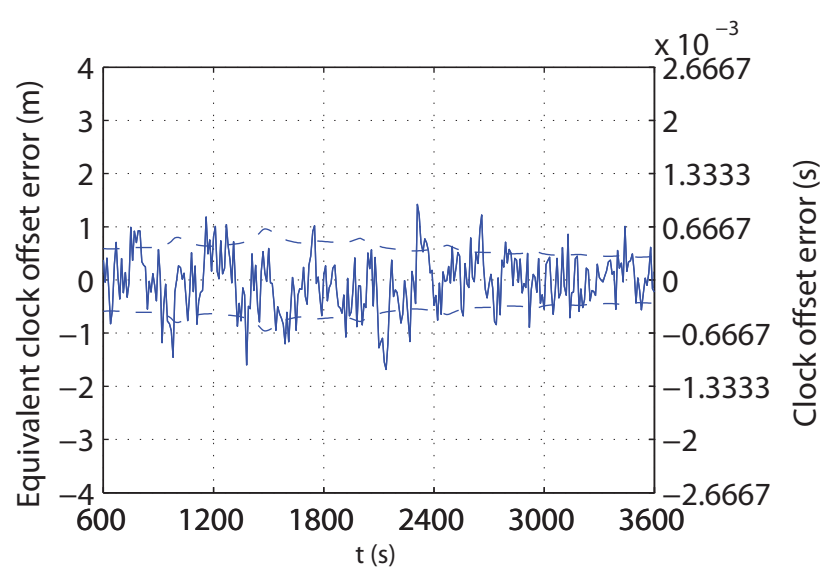

(b) Extended Kalman filter

Figure 8: Steady-state evolution of the error of the clock offset, with $3-\sigma$ bounds in dashed lines 
presence of sensor noise, otherwise it would converge to zero). The detailed evolutions of the position and velocity errors are depicted in Figs. 6(a) and 7(a), respectively, along with the 3- $\sigma$ bounds. The most noticeable feature is that the position and velocity errors remain, most of the time, below $1 \mathrm{~m}$ and $0.01 \mathrm{~m} / \mathrm{s}$, respectively. The detailed evolution of the error related to the clock offset is shown in Fig. 8(a), along with the $3-\sigma$ bounds. As it is possible to observe, the proposed solution effectively estimates the clock offset, whose error remains, most of the time, below $2 \mathrm{~m}$. For the sake of completeness, the evolution of the differences of the pseudo-range errors is shown in Fig. 9 and the evolution of the norm of the complete estimation error vector is shown in Fig. 10.

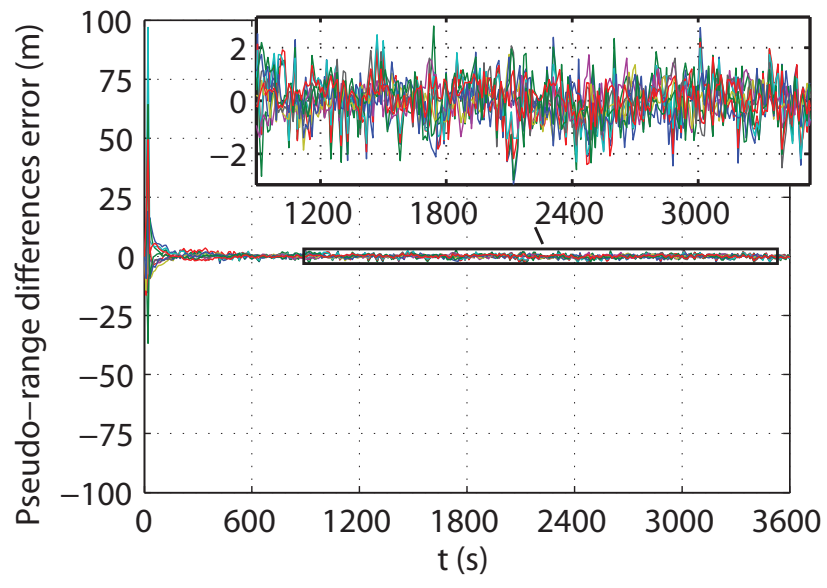

Figure 9: Evolution of the errors of the pseudo-range differences

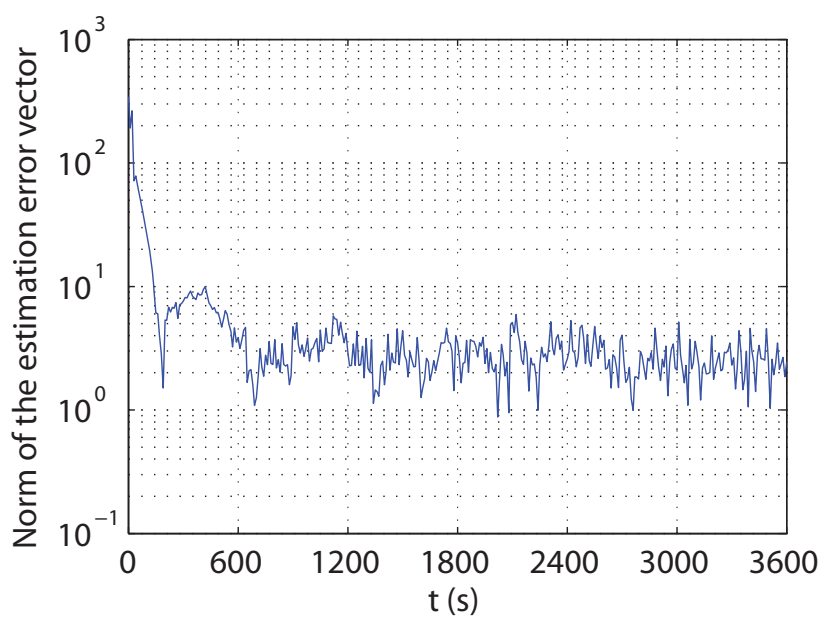

Figure 10: Evolution of the norm of the estimation error vector

\subsection{Performance comparison}

The proposed solution was compared with the EKF applied to the original nonlinear system (5). The initial estimates were set as in the previous simulation, only now the states that are considered are only the position, the ocean current velocity, and the factor that accounts for the clock offset. The state disturbance matrix was set to $\operatorname{diag}\left(10^{-2} \mathbf{I}, 10^{-6} \mathbf{I}, 10^{-1}\right)$ and the output noise covariance matrix was set to $0.1 \mathbf{I}$.

The initial convergence of the position and velocity errors is depicted in Figs. 3(b) and 4(b), respectively. The initial convergence of the clock offset error is shown 5(b). In comparison with the proposed solution, there are no significant differences in terms of initial transients: the EKF is slower and the initial transients are also larger for the position and ocean current velocity. The detailed evolutions of the position and velocity errors are depicted in Figs. 6(b) and 7(b), respectively, whereas the detailed evolution of the steady-state clock offset error is depicted in Fig. 8(b). The EKF performs, in steady-state, similarly to the proposed solution. It does not offer, however, global convergence guarantees.

Finally, in order to better evaluate the performance of the proposed solution, the Monte Carlo method was applied, and 1000 simulations were carried out with different, randomly generated noise signals. The standard deviation of the steady-state errors were computed for each simulation and averaged over the set of simulations. The results are depicted in Table 1. The results with the EKF are also included. As it is possible to observe, both solutions achieve close performances. The main advantage of the novel solution proposed here is that it guarantees global convergence.

Table 1: Standard deviation of the steady-state estimation error, averaged over 1000 runs of the simulation

\begin{tabular}{c|c|c}
\hline Variable & Standard deviation & EKF standard deviation \\
\hline$\tilde{\mathbf{p}}_{x}(\mathrm{~m})$ & $66.4 \times 10^{-2}$ & $48.1 \times 10^{-2}$ \\
$\tilde{\mathbf{p}}(\mathrm{m})$ & $99.7 \times 10^{-2}$ & $75.4 \times 10^{-2}$ \\
$\tilde{\mathbf{p}}_{z}(\mathrm{~m})$ & $77.6 \times 10^{-2}$ & $58.6 \times 10^{-2}$ \\
$\tilde{\mathbf{v}}_{c x}(\mathrm{~m} / \mathrm{s})$ & $3.10 \times 10^{-3}$ & $4.46 \times 10^{-3}$ \\
$\tilde{\mathbf{v}}_{c y}(\mathrm{~m} / \mathrm{s})$ & $3.95 \times 10^{-3}$ & $5.54 \times 10^{-3}$ \\
$\tilde{\mathbf{v}}_{c z}(\mathrm{~m} / \mathrm{s})$ & $3.24 \times 10^{-3}$ & $4.60 \times 10^{-3}$ \\
$b_{c}(\mathrm{~m})$ & 1.31 & 0.56 \\
\hline
\end{tabular}

\subsection{Slowly time-varying clock offset}

The clock offset was assumed, in nominal terms, as constant in this paper. Nevertheless, considering that it is driven by a Gaussian white process, within a Kalman filtering framework, it is actually possible to track slowly time-varying clock offsets by appropriate tunning of the Kalman filter. In order to illustrate this scenario, the simulation presented in Section 4.1 was modified and a constant clock drift is here considered. In particular, the term that accounts of the clock offset starts with $b_{c}(0)=50 \mathrm{~m}$ and after one hour it is at $100 \mathrm{~m}$, changing linearly in-between, which corresponds to a constant clock drift of roughly 0.8 seconds per day. In terms of convergence speed or the magnitude of the initial transients there are no visible changes. The detailed evolutions of the position and the inertial ocean current velocity are depicted in Figs. 11 and 12, whereas the estimation error of the term that accounts for the clock offset is depicted in Fig. 13. From these plots it is possible to conclude that the proposed solution in indeed able to cope well 
with slowly time-varying clock offsets, with only a small degradation of the estimation performance.

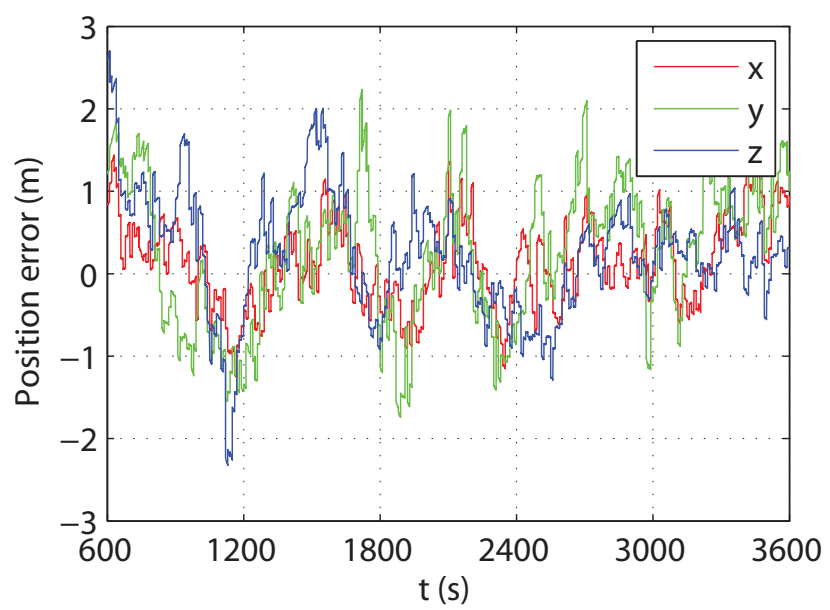

Figure 11: Steady-state evolution of the position error with a slowly timevarying clock offset

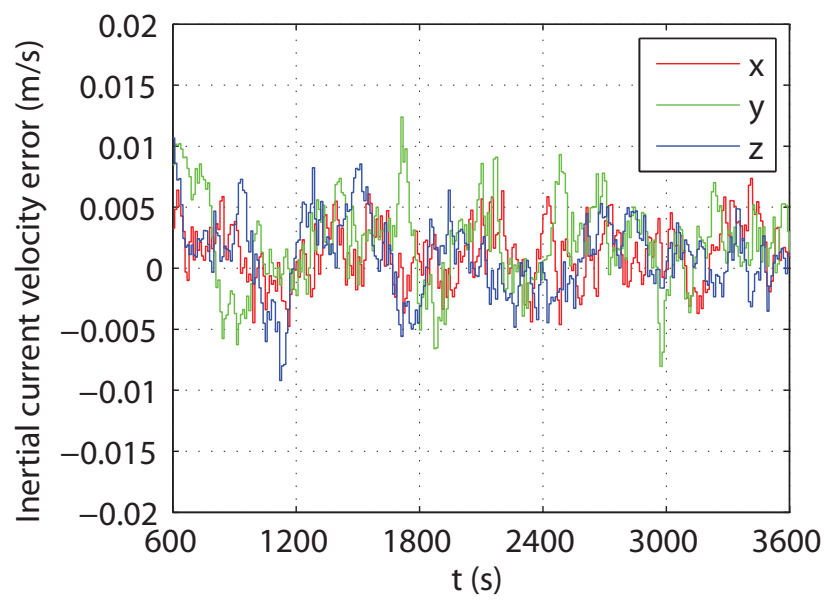

Figure 12: Steady-state evolution of the ocean current velocity error with a slowly time-varying clock offset

\section{Conclusions}

LBL navigation systems that operate in OWTT mode typically require the clock synchronization between the acoustic beacons fixed in the mission scenario and the acoustic receiver installed on-board the vehicle. This is an additional and very heavy burden. This paper proposed a novel filtering solution that explicitly estimates a constant unknown clock offset, thus relaxing the need for clock synchronization. The observability of the system was carefully detailed, in a constructive way, such that a Kalman filter with globally exponentially stable error dynamics can be applied. The continuous-discrete nature of the problem was taken into account and simulation results, including sensor noise, were presented, evidencing good filtering performance. Additional comparison with the EKF, including Monte Carlo runs, was carried out, revealing similar performances. However, the proposed solution provides guarantees of

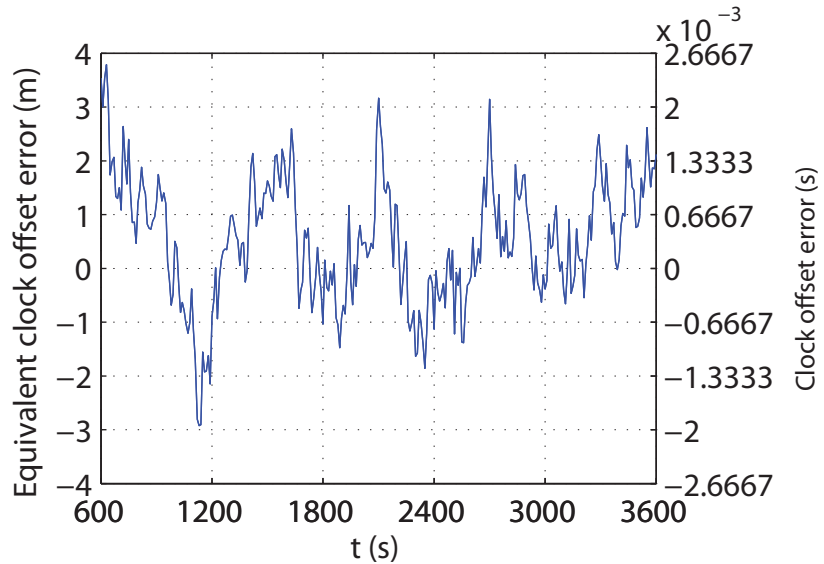

Figure 13: Steady-state evolution of the error of the clock offset, which is timevarying

global convergence, which are absent in the case of the EKF. Finally, the filter was also shown to cope well, in simulation, with a slowly time-varying clock offset. The inclusion of the estimation of the sound speed profile, or equivalent average sound speed, is an interesting topic for future work. Another interesting avenue is the incorporation of outlier rejection algorithms that work at the pseudo-range level, as those measurements are also included in the system state. An alternative but very similar setup could be envisioned by considering a constant clock drift instead of a constant clock offset, with the inclusion of an additional state corresponding to the constant clock drift. Finally, field trials are required to evaluate the proposed solution.

\section{Acknowledgements}

This work was supported by the FCT [PEstOE/EEI/LA0009/2013].

\section{References}

[1] Batista, P., Dec. 2013. GES Long Baseline Navigation with Unknown Sound Velocity and Discrete-time Range Measurements. In: Proceedings of the 52nd IEEE Conference on Decision and Control. Florence, Italy, pp. 6176-6181.

[2] Batista, P., Jun. 2014. GES Long Baseline Navigation with Clock Offset Estimation. In: Proceedings of the 2014 European Control Conference. Strasbourg, France, pp. 3011-3016.

[3] Batista, P., Silvestre, C., Oliveira, P., Aug. 2011. Single Range Aided Navigation and Source Localization: observability and filter design. Systems \& Control Letters 60 (8), 665-673.

[4] Batista, P., Silvestre, C., Oliveira, P., Jul. 2014. Sensor-based Long Baseline Navigation: observability analysis and filter design. Asian Journal of Control 16 (4), 974-994.

[5] Caffery, J., Sep. 2000. A new approach to the geometry of TOA location. In: Proceedings of the IEEE VTS Fall VTC2000 52nd Vehicular Technology Conference. Vol. 4. Boston, USA, pp. 1943-1949.

[6] Eustice, R., Whitcomb, L., Singh, H., Grund, M., Apr. 2007. Experimental Results in Synchronous-Clock One-Way-Travel-Time Acoustic Navigation for Autonomous Underwater Vehicles. In: Proceedings of the 2007 Int. Conference on Robotics and Automation. Rome, Italy, pp. 42574264 . 
[7] Gadre, A., Stilwell, D., Aug. 2005. A complete solution to underwater navigation in the presence of unknown currents based on range measurements from a single location. In: Proceedings of the 2005 IEEE/RSJ International Conference on Intelligent Robots and Systems. Edmonton AB, Canada, pp. 1420-1425.

[8] Garau, B., Alvarez, A., Olivier, G., May 2006. AUV navigation through turbulent ocean environments supported by onboard H-ADCP. In: Proceedings of the 2006 IEEE Conference on Robotic and Automation. Orlando, FL, USA, pp. 3556-3561.

[9] Hegrenas, O., Gade, K., Hagen, O., Hagen, P., Oct. 2009. Underwater transponder positioning and navigation of autonomous underwater vehicles. In: Proceedings of the 2009 MTS/IEEE OCEANS. Biloxi, USA, pp. $1-7$.

[10] Hegrens, O., Hallingstad, O., Apr. 2011. Model-Aided INS With Sea Current Estimation for Robust Underwater Navigation. IEEE Journal of Oceanic Engineering 36 (2), 316-337.

[11] Hunt, M., Marquet, W., Moller, D., Peal, K., Smith, W., Spindel, R., Dec. 1974. An acoustic navigation system. Tech. Rep. Technical Report 74-6, Woods Hole Oceanographic Institution.

[12] Jazwinski, A., 1970. Stochastic Processes and Filtering Theory. Academic Press, Inc.

[13] Jouffroy, J., Opderbecke, J., Jun-July 2004. Underwater vehicle trajectory estimation using contracting PDE-based observers. In: Proceedings of the 2004 American Control Conf. Vol. 5. Boston, MA, USA, pp. 4108-4113.

[14] Kinsey, J., Eustice, R., Whitcomb, L., Sep. 2006. A Survey of Underwater Vehicle Navigation: Recent Advances and New Challenges. In: Proceedings of the 7th IFAC Conf. on Manoeuvring and Control of Marine Craft. Lisboa, Portugal.

[15] Kinsey, J., Whitcomb, L., Dec. 2004. Preliminary field experience with the DVLNAV integrated navigation system for oceanographic submersibles. Control Engineering Practice 12 (12), 1541-1548.

[16] LaPointe, C., Jun. 2006. Virtual long baseline (VLBL) autonomous underwater vehicle navigation using a single transponder. Master's thesis, Massachusetts Institute of Technology.

[17] Larsen, M., Sep. 2000. Synthetic long baseline navigation of underwater vehicles. In: Proceedings of the 2000 MTS/IEEE OCEANS. Vol. 3. Providence, RI, USA, pp. 2043-2050.

[18] Leonard, J., Bennett, A., Smith, C., Feder, H., 1998. Autonomous underwater vehicle navigation. Tech. Rep. Technical Memorandum 98-1, MIT Marine Robotics Laboratory.

[19] Leonard, J., Rikoski, R., 2001. Incorporation of delayed decision making into stochastic mapping. In: Rus, D., Singh, S. (Eds.), Experimental Robotics VII. Vol. 271 of Lecture Notes in Control and Information Sciences. Springer Berlin Heidelberg, pp. 533-542.

[20] Milne, P., 1983. Underwater Acoustic Positioning Systems. Gulf Publishing Co.

[21] Morgado, M., Batista, P., Oliveira, P., Silvestre, C., Dec. 2011. Position USBL/DVL Sensor-based Navigation Filter in the presence of Unknown Ocean Currents. Automatica 47 (12), 2604-2614.

[22] Olson, E., Leonard, J., Teller, S., Oct. 2006. Robust Range-Only Beacon Localization. IEEE Journal of Oceanic Engineering 31 (4), 949-958.

[23] Peyronnet, J.-P., Person, R., Rybicki, F., Sept.-Oct. 1998. POSIDONIA 6000: a new long range highly accurate ultra short base line positioning system. In: Proceedings of the 1998 IEEE OCEANS Conference. Nice, France, pp. 1721-1727.

[24] Rikoski, R., Jun. 2003. Dynamic sonar perception. Ph.D. dissertation, Massachusetts Institute of Technology.

[25] Rugh, W., 1995. Linear system theory, 2nd Edition. Prentice-Hall, Inc.

[26] Stanway, M., Feb. 2012. Contributions to Automated Realtime Underwater Navigation. Ph.D. dissertation, Massachusetts Institute of Technology / Woods Hole Oceanographic Institution Joint Program.

[27] Tan, H.-P., Diamant, R., Seah, W., Waldmeyer, M., Oct. 2011. A survey of techniques and challenges in underwater localization. Ocean Engineering 38 (14-15), 1663-1676.

[28] Techy, L., Morgansen, K., Woolsey, C., Jun.-Jul. 2011. Long-baseline acoustic localization of the Seaglider underwater glider. In: Proceedings of the 2011 American Control Conference. San Francisco, USA, pp. 3990-3995.

[29] Vaganay, J., Bellingham, J., Leonard, J., Oct. 1998. Comparison of fix computation and filtering for autonomous acoustic navigation. Int. J. of Systems Science 29 (10), 1111-1122.
[30] Webster, S., Eustice, R., Singh, H., Whitcomb, L., Oct. 2009. Preliminary deep water results in single-beacon one-way-travel-time acoustic navigation for underwater vehicles. In: Proceedings of the 2009 IEEE/RSJ International Conference on Intelligent Robots and Systems- IROS 2009. SaintLouis, MO, USA, pp. 2053-2060.

[31] Webster, S., Eustice, R., Singh, H., Whitcomb, L., Jul. 2012. Advances in single-beacon one-way-travel-time acoustic navigation for underwater vehicles. The International Journal of Robotic Research 31 (8), 935-950.

[32] Whitcomb, L., Yoerger, D., Singh, H., Aug. 1999. Combined Doppler/LBL Based Navigation of Underwater Vehicles. In: Proceedings of the 11th International Symposium on Unmanned Untethered Submersible Technology. Durham, New Hampshire, USA, pp. 1-7.

[33] Yoerger, D., Jakuba, M., Bradley, A., Bingham, B., Jan. 2007. Techniques for deep sea near bottom survey using an autonomous underwater vehicle. The International Journal of Robotics Research 26 (1), 41-54.

\section{Appendix A. Dynamics of the pseudo-range differences}

To compute the dynamics of the pseudo-range differences, expand first $\left[r_{i}(k)-x_{3}(k)\right]^{2}$, which using the pseudo-range definition gives

$$
r_{i}^{2}(k)+x_{3}^{2}(k)-2 r_{i}(k) x_{3}(k)=\left\|\mathbf{x}_{1}(k)\right\|^{2}+\left\|\mathbf{s}_{i}\right\|^{2}-2 \mathbf{s}_{i} \cdot \mathbf{x}_{1}(k)
$$

for all $i \in\{1, \ldots, L\}$. Taking (A.1) at time $k+1$ and substituting the dynamics of $\mathbf{x}_{1}(k+1)$ and $x_{3}(k+1)$ gives

$$
\begin{gathered}
r_{i}^{2}(k+1)+x_{3}^{2}(k)-2 r_{i}(k+1) x_{3}(k)= \\
\left\|\mathbf{x}_{1}(k)\right\|^{2}+\left\|\mathbf{s}_{i}\right\|^{2}-2 \mathbf{s}_{i} \cdot \mathbf{x}_{1}(k) \\
-2 T \mathbf{s}_{i} \cdot \mathbf{x}_{2}(k)+2 T \mathbf{u}(k) \cdot \mathbf{x}_{2}(k)+2 T \mathbf{x}_{1}(k) \cdot \mathbf{x}_{2}(k) \\
+2 \mathbf{u}(k) \cdot \mathbf{x}_{1}(k)+T^{2}\left\|\mathbf{x}_{2}(k)\right\|^{2}-2 \mathbf{s}_{i} \cdot \mathbf{u}(k)+\|\mathbf{u}(k)\|^{2}
\end{gathered}
$$

for all $i \in\{1, \ldots, L\}$. Substituting (A.1) in (A.2) and rearranging the terms gives

$$
\begin{gathered}
r_{i}^{2}(k+1)=r_{i}^{2}(k)+2\left[r_{i}(k+1)-r_{i}(k)\right] x_{3}(k) \\
-2 T \mathbf{s}_{i} \cdot \mathbf{x}_{2}(k)+2 T \mathbf{u}(k) \cdot \mathbf{x}_{2}(k)+2 T \mathbf{x}_{1}(k) \cdot \mathbf{x}_{2}(k) \\
+2 \mathbf{u}(k) \cdot \mathbf{x}_{1}(k)+T^{2}\left\|\mathbf{x}_{2}(k)\right\|^{2}-2 \mathbf{s}_{i} \cdot \mathbf{u}(k)+\|\mathbf{u}(k)\|^{2}
\end{gathered}
$$

for all $i \in\{1, \ldots, L\}$. Now, from (A.3) one can write

$$
\begin{gathered}
r_{i}^{2}(k+1)-r_{j}^{2}(k+1)=r_{i}^{2}(k)-r_{j}^{2}(k) \\
+2\left[r_{i}(k+1)-r_{i}(k)\right] x_{3}(k)-2\left[r_{j}(k+1)-r_{j}(k)\right] x_{3}(k) \\
-2 T\left[\mathbf{s}_{i}-\mathbf{s}_{j}\right] \cdot \mathbf{x}_{2}(k)-2\left[\mathbf{s}_{i}-\mathbf{s}_{j}\right] \cdot \mathbf{u}(k)
\end{gathered}
$$

for all $i, j \in\{1, \ldots, L\}, i \neq j$. Finally, using $a^{2}-b^{2}=(a+$ $b)(a-b)$ in (A.4) twice gives (8). 